\title{
A Categorization Scheme for Semantic Web Search Engines
}

\author{
Kyumars Sheykh Esmaili, Hassan Abolhassani \\ Semantic Web Research Laboratory \\ Computer Engineering Department \\ Sharif University of Technology, Tehran, Iran \\ shesmail@ce.sharif.edu, abolhassani@sharif.edu
}

\begin{abstract}
Semantic web search engines are evolving and many prototype systems and some implementation have been developed. However, there are some different views on what a semantic search engine should do. In this paper, a categorization scheme for semantic web search engines are introduced and elaborated. For each category, its components are described according to a proposed general architecture and various approaches employed in these components are discussed. We also propose some factors to evaluate systems in each category.
\end{abstract}

\section{INTRODUCTION}

This paper tries to analysis semantic search engines and provides a rational categorization scheme for them. To the best of our knowledge, in this regards there is no work reported. However, there is only a short explanation in [19].

According to [5], semantic web (hereafter is referred to as $\mathrm{SW}$ ) has some distinguishing features that affects search process:

- Instead of web documents, in the SW, all objects of the real world are involved in the search.

- Information in SW is understandable by machines as well as human.

- SW languages are more advanced than html.

- It is possible to distribute information about a single concept in SW.

Therefore semantic search engines have following fundamental differences to the traditional search engines:

- Using a logical framework lets more intelligent retrieval possible.

- There are more complex relations in documents resulting in the importance of the problem of meta-data maintenance, update and more complex ranking.

- Specifying relationships among objects explicitly highlights the need for better visualization techniques for the results of a search.

One important aspect of SW search is the usage of ontology and meta-data. Ontology provides explicit conceptualization for entities in a specific domain. Another important aspect is the annotation for the current web pages. Annotations are meta-data useful for machines to understand the content of a web page. In such meta-data concepts are pointers to already defined ontologies [24]. Respecting the kind of search in SW, it is possible to categorize users to two groups. One group are ordinary users that do searching as like the current web but demand more accurate and complete results than traditional search engines. Second group are application developers in the SW where their primary goal is to search and retrieve SW documents. According to these two categories of users, we can categorize SW search engines to the following two categories:

- Engines specific to the SW documents: they search only documents that are represented in one of the languages specific to SW.

- Engines that tries to improve search results using SW standards and languages. Using context information (represented by domain ontology and metdata) is one of the key aspects for these engines.

The paper is organized as follows. In section 2 different types of annotation and methods of generating them are discussed. Section 3 provides explanations about search engines for developers and advanced users in SW. Search engines that use SW concepts to provide better search results are discussed and further categorized in section 4. For each category, in section 5, we have a brief analysis and propose some points for evaluating them. Section 6 concludes the paper by providing a summary of all reported works in SW search.

\section{Annotation Methods}

One of the major problems facing researchers in SW is annotation which is a prerequisite for SW search engines. To adopt current web pages for SW search engines they should be annotated by finding appropriate meta-data to be added to each one. There are different approaches which spawn in a broad spectrum from complete manual to full automatic methods. Selection of an appropriate method depends on the domain of interest [24]. In general meta-data generation for structured data is simpler [24].

Annotations can be categorized based on following aspects:

- Type of meta-data: According to [25] meta-data can be divided to two types of Structural and Semantic. In the former, non contextual information about content is expressed (e.g. language and format). In the later, the main concern is on the detailed content of information and usually is stored as RDF triples.

- Generation approach: a simple approach is to generate meta-data without considering the overall theme of the page and only using structural information of a page 
together with natural language processing techniques. A better approach is to use an ontology in the generation process. In this case it is possible to use clustering methods to distinguish the general type of a page [25]. Then using a previously specified ontology for that type, generate meta-data that instantiates concepts and relations of ontology for that page. The main advantage of this method is the usage of contextual information.

- Source of generation. The ordinary source of meta-data generation is a page itself but sometimes it is beneficial to use other complementary sources. For example [1] and [5] discusses about using network available resources for accumulating more information for a page. For example for a movie it might be possible to use IMDB to extract additional information like director, genre, etc.

Although there is no complete reference about meta-data but [18] provides a rather complete list of systems that generate meta-data.

\section{Ontology SEARCH Engines}

According to [1] and [8], for the following reasons, it is not possible to use current search engines for SW documents:

- Current techniques does not let to index and retrieve semantic tags.

- They don't use the meaning of tags

- Can't display results in visual form

- Ontologies are not separated entities which usually have cross references that current engines don't process.

In general there are two approaches to handle these documents: using current search engines with some modifications or creating a special search engine. In what follows each of these approaches is further elaborated.

\section{A. Ontology Meta Search Engines}

This group do retrieval by putting a system on top of a current search engine. There are two types of this systems. In the first type, there is a search engine that only searches specific file types (e.g. RSS, RDF, OWL). The main concerns of such systems are on the visualization and browsing of results. For example in [8] an engine forwards a users request for a specific file type to Google search engine and then using a visualization tool lets user to navigate and display results.

In the second type there is possible to search on semantic tags. But since those tags are ignored by the underlying search engine, an intermediate format for documents and user queries are used. In [2] a technique named Swangle is used for this purpose. With this technique RDF triples are translated into strings suitable for underlying search engine. For example consider the following triple which is in $n 3$ notation.

j00255 owlir:moviegenre "action".

It is translated to the following seven terms: j00255.owlir.umbc.edu/event/moviegenre.action .owlir.umbc.edu/event/moviegenre.action

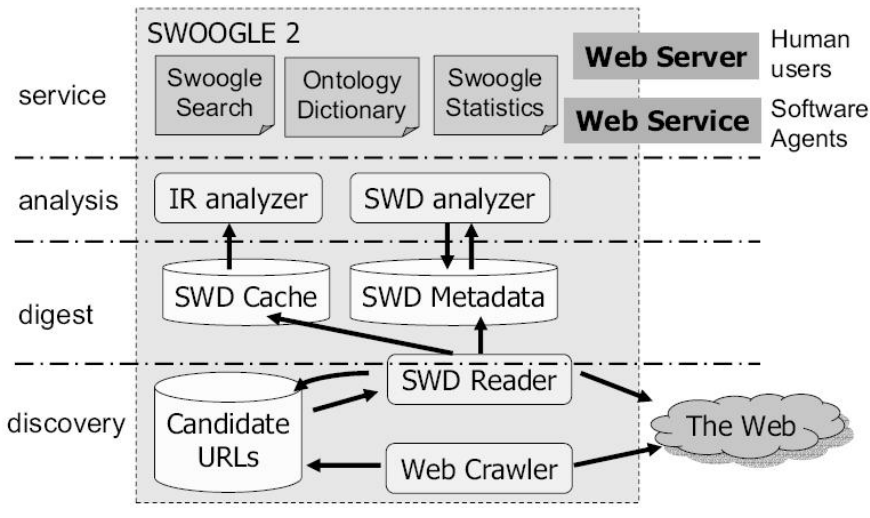

Fig. 1. Swoogle Architecture - A Sample Crawler Based Ontology Search Engine

j00255.*.action

j00255.owlir.umbc.edu/event/moviegenre.*

j00255.***

.owlir.umbc.edu/event/moviegenre.*

*.action

Each of these terms are converted to a string and added to the document for indexing. On the other side, this translation is done for user queries too.

\section{B. Crawler Based Ontology Search Engines}

These engines uses a specific crawler for SW documents. In the figure 1 one complete system is shown[4]: Architecture of a SW document specific search engine

Here, based on the four sections specified in the architecture, analysis of them is given.

1) Discovery: Crawling of SW documents is different from html documents. Actually they are knowledge crawlers which are more complex than traditional ones [27]. In SW we express knowledge using URI in RDF triples. Unlike html hyperlinks, URIs in RDF may point to a non existing entity. Also RDF may be embedded in html documents or be stored in a separate file.

Such crawlers should have the following properties [27]:

- Should crawl on heterogeneous web resources (owl, oil, daml, rdf, xml, html) .

- Avoid circular links

- Completing RDF holes

- Finding new semantic web documents from information in the currently under process document (e.g. Extend and Import specifications).

In [26] crawling of SW documents is explained in detail. According to [27] it is possible to categorize the derived ontologies based on a clustering method. For indexing and retrieval of SW documents $\mathrm{N}$-grams and bag of URI refs are proposed. More explanation and comparison of them are given in [3].

Three types of meta data are used in these systems [3]: 
- Language attributes

- Relationships between SW documents (prior version, imports, extends, etc.)

- Meta data resulting from analysis

2) Analysis: For offline ranking it is possible to use the references idea of PageRank. But the main point is that count, type and meaning of relations in SW is more complete than the current web. In the table I three types of relation and their corresponding values are specified[27]:

In [4] OntoRank values for each ontology is calculated very similar to PageRank in traditional search engines like google.

\begin{tabular}{|c|c|c|}
\hline Priority & Relationship & Language Specific \\
\hline 1 & instantiation & rdf:type \\
2 & subClass & rdfs:subClass, daml:subClass \\
3 & domain/range & rdfs:domain, daml:range \\
\hline
\end{tabular}

TABLE I

RELATIONSHIP TYPES

3) Digest: In a recent version this algorithm is similar to what is given in [27] which uses a sum of rank and priority of concepts in a SW document to calculate the overall rank of a document.

4) Service: In addition to user interface in this section, services to application systems are provided too.

\section{Semantic Search Engines}

Searching in the web is done either using search engines or web directories, each having respected restrictions [5]. One interesting example is shown in [5]: if we search for "Matrix", non homogeneous results ranging from mathematical matrix, Matrix movie and so on is returned. Semantic web is introduced to overcome such problems.

The most important tool in semantic web for improving search results is context concept and its correspondence with Ontologies. This type of search engines uses such ontological definitions.

It is possible to categorize this type of search engines to three groups. In the first group which is the largest one, aim is to add semantic operations for better results. In the second group, using facilities of semantic web the goal is to accumulate information on a topic we are researching on. Search engines in the third group try to find semantic relations between two or more terms.

\section{A. Context based Search Engines}

Figure 2 shows an overall framework for this kind of engines. It should be emphasized that very limited number of engines have all of the functionalities specified in the figure.
1) Crawling the semantic web: There is not much difference between these crawlers and ordinary web crawlers and in fact many of the implemented systems uses an existing web crawler as underlying system. For example in [1] haircut is used as underlying system and also [15] uses one that understands special semantic tags. One of the important features of theses crawlers should be the exploration of ontologies that are referred from existing web pages.

2) Metadata generation: According to discussion in section 3, there are different ways for metadata generation. For example [1] and [5] use external metadata. [1] Uses AeroText to extract names and expressions and then generates metadata in RDF format. One of the important problems in this regards is semantic normalization [25] meaning to generate metadata for different resources in same form. For example [12] is a non-standard example in which metadata is represented in ad hoc model.

In semantic portal [23] producers should generate annotations and there is a summarization and collection of metadata in the central server.

As explained before, metadata generation is simpler and more accurate when the theme of a page is known. For example in [15] using a tool named Knowledge Annotator terms of ontology is used to describe information in a given page. Also [18] proposed a method for generating and managing metadata according to already defined ontologies.

But if ontology for a page is not known in advance, it is possible to use clustering techniques like what explained in [25] to find an appropriate ontology. Knowledge Parser [24] is a kind of complete system using important techniques from different areas like NLP, Text Engineering, Document Structure Processing, and Layout Processing. Its operation is shown in the figure.3.

3) Indexing: Most of the engines does not provide any special functionality regarding indexing. OWLIR [1] uses Swangling explained earlier. [6] Introduces Ontological Indexing in which indexing is done based on a reference ontology. Also in [18] possibility of dividing documents to smaller parts is used to improve indexing performance. Also in $\mathrm{p} 2 \mathrm{p}$ architecture of [22] for each of concepts in the reference ontology there exist an agent that maintains information corresponding to it.

4) Accepting user's requests: There are two different approaches: term-based and form-based. In term-based approach used in [5], [23], and [24], it is tried to find the search context from entered keywords. In the form-based approach used in [1], [15], [23], and [24], user interface is generated according to the ontology selected by user.

5) Generating meta data for user requests: This operation is very similar to generating metadata for documents. For example in [18] the same Semantic Mapper is used for generating metadata both for documents and user requests. Often Wordnet is used to expand user requests. For example in [20] for termed entered by a user, using Wordnet, synonyms is found and used to expand the query.

6) Retrieval and ranking model: Usually an ordinary VSM model [30] is used and then based on RDF graph matching 


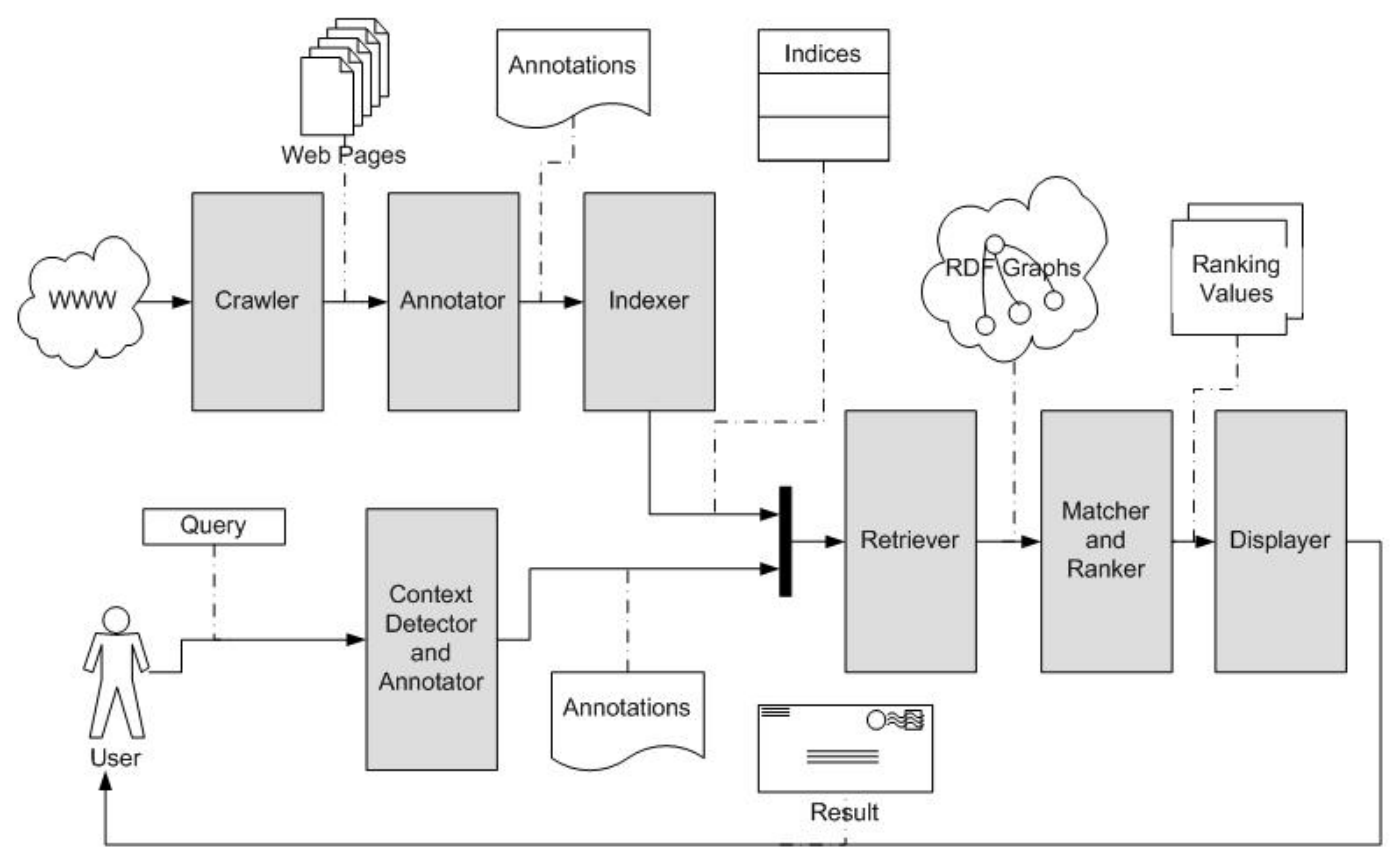

Fig. 2. Semantic Search Engines' Architecture
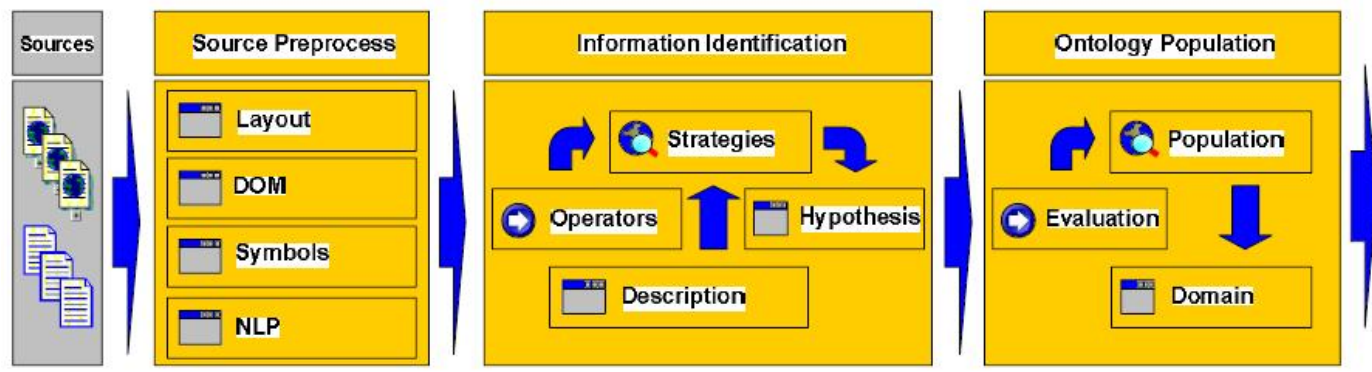

Fig. 3. Annotation Generation Steps in KnowledgeParser

results are pruned. In [9] from the equivalence of RDF graphs and Conceptual Graphs (CG), already existing operations on CGs is used to match user request and documents.

Semantic Distance concept is often used to estimate similarity of concepts in a matching process. In [21] this measure is defined for different elements in graphical representations.

It is also possible to use graph similarity for ranking results. However, in [7] a fuzzy approach is used for this purpose.

7) Display of results: A major difference of semantic search engines and ordinary ones is the display of results. One of the primary tasks is to filter the results (for example for eliminating repetitions). In [6] in addition to normal display of results, a number of classes is displayed and when a user selects one, only those results having instances of the classes is shown. In [23] display is a kind of hierarchy in which top concepts of ontology is shown and by selecting one, detail of it according to the ontology is displayed.

\section{B. Evolutionary Search Engines}

The advanced type of search is some thing like research; in fact as mentioned in [5] this kind of searches aim at gathering some information about specific topic. For example if we give the name of a singer to the search engine it should be able to find some related data to this singer like biography, posters, albums and so on. These engines usually use one of the commercial search engines as their base component for searching and then augment returned result by these base engines. This augmented information is gathered from some data-insensitive web resources. In figure 4 we showed overall architecture for such engines.

As it can be deduced from the figure this architecture has some similarities with what we discussed in previous subsection; here we crawl and generate annotation just for some well known informational web pages i.e. CDNow, Amazon, IMDB as mentioned in [2] and [5]. After this phase we collect annotations in a repository. Whenever a sample user posed a query two processes must be performed: first, we should give this query to a usual search engine (usually Google) to 


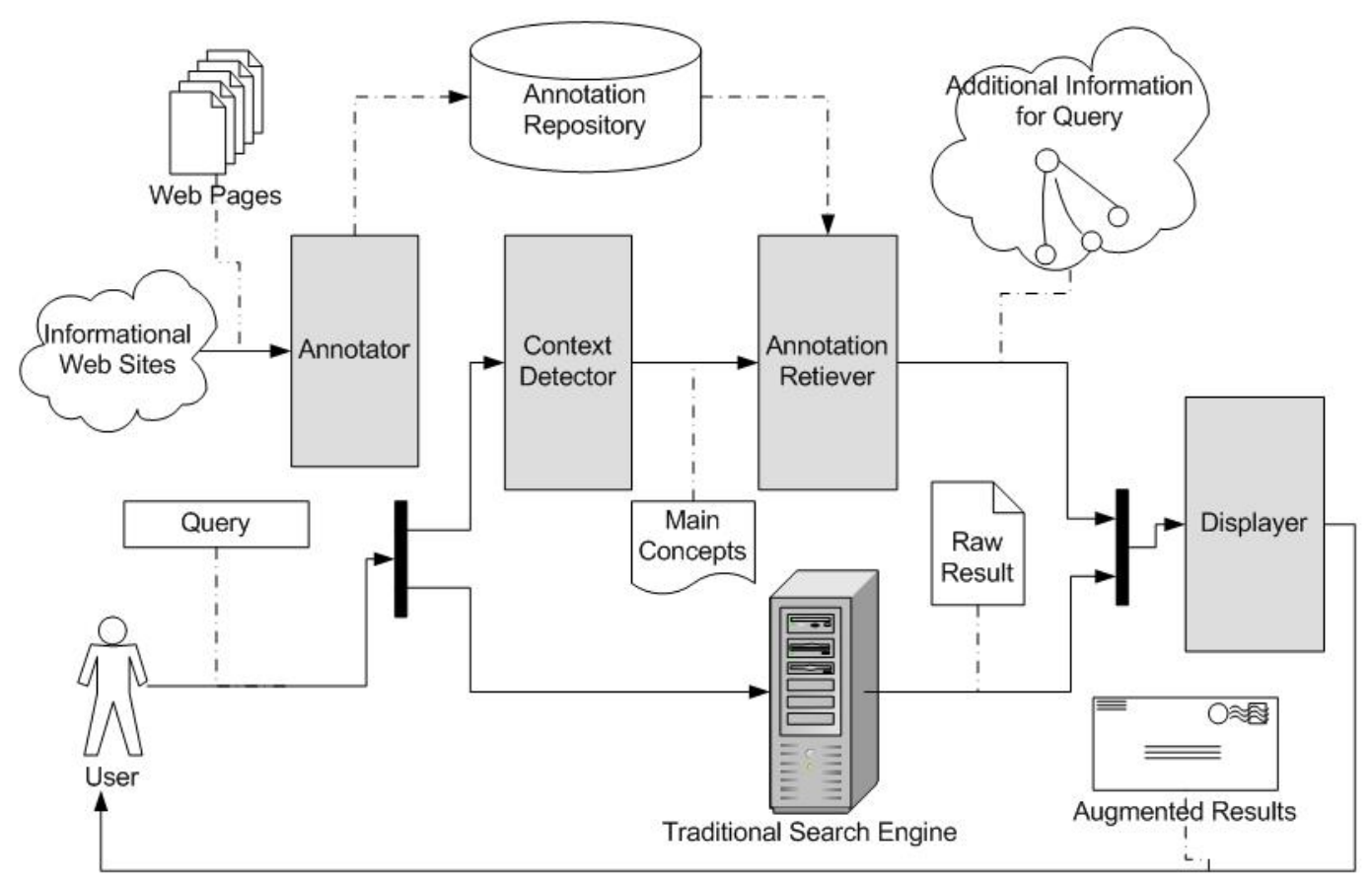

Fig. 4. Evolutionary Search Engines' Architecture

obtaining raw results. Second, system will attempt to detect the context and its corresponding ontology for the user's request in order to extract some key concepts. Later we use these concepts to fetch some information from our metadata repository. The last step in this architecture is combining and displaying results. Main problems and challenge in these types of engines are [5]:

- Concept extraction from user's request: there are some problems that lead to misunderstanding of input query by system; for example inherent ambiguity in query specified by user or complex terms that must be decomposed to be understood.

- Selecting proper annotation to display and their order: often we find a huge number of potential metadata related to the initial request and we should choose those ones that are more useful for user. A simple approach is using other concepts around our core concept (which we extracted it before) in base ontology and if we have more than one core concept we must focus on those concepts that are on the path between those core concepts.

\section{Semantic Association Discovery Search Engines}

Usually one of the user's interests is finding semantic relations between two input terms. Old search engines handled these request using learning and statistical methods [25], but semantic web standards and languages have provided more effective and precise methods. SemDis [10],[14] is a real sample for these systems, its goals is finding and ranking semantic associations. Overall architecture of SemDis is shown in figure 5. There are different types of semantic association but most known of them is a sequences of classes and relations

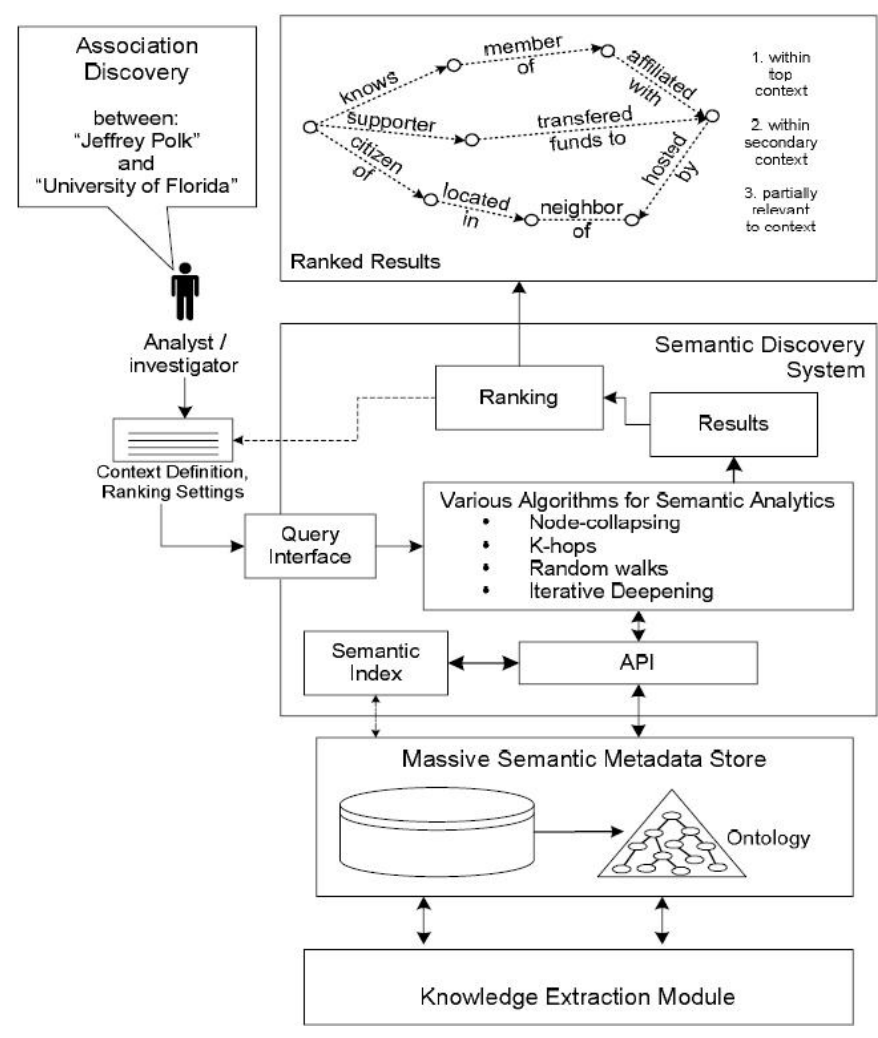

Fig. 5. SemDis Architecture - A Sample Semantic Association Discovery Engine 
between two classes. In fact we talk about just two terms because as said in [13] average length for users' queries is 2.3 term. With respect to our definition for semantic association, two terms may have one of these association: Null (both of them are instances of one concept), Direct (when there is a direct relation between them) and Indirect (chain of relations instead of single direct one). In the [13] Bayesian networks was applied in order to discover semantic association. Our reference ontology forms the graph of this network and logs of user's queries are used to computing its parameters. In general manner, for finding semantic association between more than two terms some techniques have been proposed, for example in [16] Spread Activation Technique is used to expand an initial set of instances to contain most relative instances to them. The initial set is populated by extracting important terms from user's query, then with respect to the metadata repository corresponding instances is retrieved and after expanding them an instances graph is produced which each of its edges has correctness weight in addition to usual semantic label. Technically speaking, after discovery phase often we have numerous semantic association, therefore a ranking policy must be used. In [10] some criteria for these ranking algorithms are introduced:

- Context: special part of reference ontology that is interested by user

- Subsumption: low level classes in hierarchy have more information then their parents

- Path Length: having a shorter path between two terms indicates that they have near meaning

- Trust: obtained results from trusted resources is more valuable in final ranking results

\section{Discussion And Evaluation}

Unfortunately most of these search engines has been implemented through the research projects and therefore they are not available for testing and evaluating. In the other hand because of their differences with traditional search engines it's not possible to compare them using a unique evaluation framework. Here we mention some points and hints for comparing and evaluating these search engines based on our categorization scheme presented here.

\section{A. Ontology Search Engines}

In contrast to usefulness of meta-search engines for regular pages in traditional web, it seems that they are not so good for ontologies. In fact we can not collect the all ontologies in the web just but using filetype command within commercial search engines. In addition swangling operation has a huge amount of overhead, therefore it's much better to use crawler-based ontology search engines (2nd category) rather than ontology meta-search engines (1st category). In order to evaluating performance of this kind of search engines there is no standard test collection, but we can simply test them by searching for ontologies using name of ontologies, classes and properties and judge their results according to the precision measure (portion of relevant result from all result returned)

\section{B. Semantic Search Engines}

1) Context-Based Semantic Search Engines: The main strangeness of these engines is their simplicity. In fact because they tried to be as simple as textbox search engines (like google) they are most popular search engines in the semantic web. Here quality of results heavily depends on power of its annotation module. The biggest problem of these search engines is that they are limited to the special contexts. It is much better if we can develop a multi-contextual semantic search engine. Fortunately we can apply standard measures (i.e. Precision and Recall) and standard test collections (i.e. TREC tracks) of traditional information retrieval to evaluate this kind of semantic web search engines. It should be noted that if we can prepare ontology for test documents, the results will show much improvements.

2) Evolutionary Semantic Search Engines: Main purpose of this type of search engines is information gathering for user's request. We can treat these engines as the semantic type of HITS-based search engines (i.e. Teoma) which exploit hub and authority pages for user's request. This category of search engines is usually specific for special application domains and if we want to scale them up to the whole web, we must annotate all of the web pages, therefore it leads to a set of context-based semantic search engines (previous category)

3) Semantic Association Discovery Engines: Compared to other categories, the semantic association discovery engines are related to higher layers of semantic web cake (logic and proof). Result of these engines is very depending on their ontology repository. For evaluating them we can use an upper ontology like WordNet, after selecting two concepts randomly, the correctness and speed of discovering paths between them are two useful measures for performance evaluation.

\section{CONCLUSION}

In this paper we tried to provide a categorization scheme for different types of search engines on the semantic web. We summarized our discussionsthis in Table II.

It should be noted that some of these engines are constructed through a portal and therefore they have not individual names so we mentioned name of their containing portal instead. In addition for those ones that they have not identifying name we mentioned just their reference paper.

\section{FUTURE WORK}

One of the important branches in the context of information retrieval is multimedia information retrieval. Here we only focused on the text retrieval so multimedia information retrieval on the semantic web is a new area for research. Knowing that these kind of search engines strongly depend on annotation, It seems that methods of generating metadata for multimedia documents is the most important part of these engines. Currently there are many active research projects concentrated on annotation generation methods, as explained before, this process is a vital perquisite for intelligent search, therefore, we need a practical taxonomy for these methods. Another useful work in this area is preparing standard test collection 


\begin{tabular}{|c|c|c|c|c|}
\hline \multicolumn{5}{|c|}{ Semantic Web Search Engines } \\
\hline \multicolumn{2}{|c|}{ Ontology Search Engines } & \multicolumn{3}{|c|}{ Semantic Search Engines } \\
\hline $\begin{array}{c}\text { Ontology Meta Search } \\
\text { Engines }\end{array}$ & $\begin{array}{l}\text { Crawler Based Ontology } \\
\text { Search Engines }\end{array}$ & Context Based Search Engines & $\begin{array}{c}\text { Evolutionary Search } \\
\text { Engines }\end{array}$ & $\begin{array}{l}\text { Semantic Association } \\
\text { Discovery Engines }\end{array}$ \\
\hline $\begin{array}{l}\text { Here we want to } \\
\text { find SWDs specially } \\
\text { ontologies. There is two } \\
\text { approach in using usual } \\
\text { search engines: search } \\
\text { only by the name of files } \\
\text { and use some options like } \\
\text { filtetype (rdf,owl,rss,..) } \\
\text { or search by labels } \\
\text { by converting both } \\
\text { documents and queries to } \\
\text { intermediate format that is } \\
\text { not ignorable for ordinary } \\
\text { search engines. In this } \\
\text { type of search engine } \\
\text { having a good display } \\
\text { module for browsing and } \\
\text { navigating the founded } \\
\text { ontologies is critical point }\end{array}$ & $\begin{array}{l}\text { Applications of these search } \\
\text { engines are like to the per- } \\
\text { vious category. But here } \\
\text { we use a specific crawler } \\
\text { for finding SWDs on the } \\
\text { web, index them and extract } \\
\text { some metadata about them. } \\
\text { By using these engines we } \\
\text { can search be special class } \\
\text { or property and even for } \\
\text { sample data (ABox). Graph } \\
\text { structure of the SWDs on } \\
\text { the web can be explored by } \\
\text { use of these search engines. } \\
\text { Also here visualizing the re- } \\
\text { sults is important. Preparing } \\
\text { a standard test collection for } \\
\text { these engines is a challeng- } \\
\text { ing problem. }\end{array}$ & $\begin{array}{l}\text { The final purpose of these engines is } \\
\text { enhancing performance of traditional } \\
\text { search engines (especially Precision } \\
\text { and Recall). It's possible through un- } \\
\text { derstanding the context of documents } \\
\text { and queries. One of the most important } \\
\text { part of this type is annotator which } \\
\text { responsible for generating metadata for } \\
\text { crawled pages. We need to generate } \\
\text { some metadata for user's query in or- } \\
\text { der to detect its context. Here usually } \\
\text { after traditional retrieval we combine } \\
\text { matching RDF graphs to obtain better } \\
\text { quality of results. these engine are the } \\
\text { most practical ones, in fact they are } \\
\text { the next generation of current search } \\
\text { engines. We can evaluate them using } \\
\text { traditional performance measures and } \\
\text { test collections }\end{array}$ & $\begin{array}{l}\text { This search engine is an } \\
\text { answer to a very fa- } \\
\text { mous well known prob- } \\
\text { lem: automatically gath- } \\
\text { ering information on the } \\
\text { one specific topic. The } \\
\text { main distinguished be- } \\
\text { havior of this engine is } \\
\text { using external metadata. } \\
\text { They usually use an or- } \\
\text { dinary search engine and } \\
\text { display augmented in- } \\
\text { formation near the origi- } \\
\text { nal results. We think that } \\
\text { in a large-scale mode } \\
\text { like (i.e. in whole web) } \\
\text { they will be very similar } \\
\text { to a multi context based } \\
\text { search engines }\end{array}$ & $\begin{array}{l}\text { There is a specific } \\
\text { application of the } \\
\text { semantic web for the } \\
\text { search capabilities. } \\
\text { The goal is finding } \\
\text { various semantic } \\
\text { relations between } \\
\text { input terms (usually } \\
\text { two) and then rank } \\
\text { the results based on } \\
\text { semantic distances } \\
\text { metrics. They work } \\
\text { better in the context } \\
\text { of Knowledge Bases. } \\
\text { An upper ontology } \\
\text { like WordNet or } \\
\text { OpenCyc can be } \\
\text { used for evaluating } \\
\text { this kind of search } \\
\text { engines }\end{array}$ \\
\hline $\begin{array}{l}\text { Swangler[2], } \\
\text { OntoSearch[8] }\end{array}$ & $\begin{array}{l}\text { Swoogle }[2,3,4], \\
\text { Ontokhoj[27] }\end{array}$ & $\begin{array}{l}\text { OWLIR[2], } \\
\text { QuizRDF[6], InWiss[7], } \\
\text { Dorese[9], Infofox[12], }\end{array}$ & $\begin{array}{c}\text { W3C Semantic } \\
\text { Search[5], ABC[5] }\end{array}$ & $\begin{array}{c}\text { SemDis }[10,14], 13, \\
16\end{array}$ \\
\hline
\end{tabular}

TABLE II

SUMMERY OF CATEGORIZATION

for evaluating ontology search engines. For enterprise search engines on the semantic web two important issues arise. In one hand, we need a good collection of ontologies. Based on this requirement constructing ontologies for web is a necessary task, however, using information retrieval for this purpose as mentioned in [29] is a challenging problem. On the other hand automatic context detection based on user's query is another open problem.

\section{ACKNOWLEDGMENT}

This paper is a partially founded by Sharif Semantic Web project. The authors like to thank their colleagues in Semantic Web Lab, specially Yasser Ganji Saffar and Salman MirGhasemi.

\section{REFERENCES}

[1] J. Mayfield, T. Finin, and B. County, "Information retrieval on the semantic web: Integrating inference and retrieval," in SIGIR Workshop on the Semantic Web, Toronto, Canada, 2004.

[2] T. Finin, J. Mayfield, C. Fink, A. Joshi, and R. S. Cost, "Information retrieval and the semantic web," in Proceedings of the 38th International Conference on System Sciences, Hawaii, United States of America, 2005.

[3] L. Ding, T. Finin, A. Joshi, R. Pan, R. S. Cost, Y. Peng, P. Reddivari, V. C. Doshi, and J. Sachs, "Swoogle: A search and metadata engine for the semantic web," in Proceedings of the Thirteenth ACM Conference on Information and Knowledge Management, 2004.

[4] T. Finin, L. Ding, R. Pan, A. Joshi, P. Kolari, A. Java, and Y. Peng, "Swoogle: Searching for knowledge on the semantic web," in Proceedings of the AAAI 05, 2005.

[5] R. Guha, R. McCool, and E. Miller, "Semantic search," in Proc. of the 12th international conference on World Wide Web, New Orleans, 2003, pp. 700-709.
[6] J. Davies, R. Weeks, and U. Krohn, "Quizrdf: Search technology for the semantic web," in WWW2002 Workshop on RDF and Semantic Web Applications, 2002.

[7] T. Priebe, C. Schlaeger, and G. Pernul, "A search engine for RDF metadata," in Proc. of the DEXA 2004 Workshop on Web Semantics, 2004.

[8] Y. Zhang, W. Vasconcelos, and D. Sleeman, "OntoSearch: An ontology search engine," in The Twenty-fourth SGAI International Conference on Innovative Techniques and Applications of Artificial Intelligence, Cambridge, 2004.

[9] O. Corby, R. Dieng-Kuntz, and C. Faron-Zucker, "Querying the semantic web with the corese search engine," in Proc. 15th ECAI/PAIS, Valencia, Spain, 2004.

[10] C. Halaschek, B. Aleman-Meza, I. Arpinar, and A. Sheth, "Discovering and ranking semantic associations over a large RDF metabase," in 30th International Conference on Very Large Data Bases(VLDB), Toronto, Canada, 2004.

[11] H. Yu, T. Mine, and M. Amamiya, "An architecture for personal semantic web information retrieval system," in 14th international conference on World Wide Web table of contents, Chiba, Japan, 2005.

[12] B. Sigrist and P. Schubert, "From full text search to semantic web: The Infofox project," in Proceedings of the Tenth Research Symposium on Emerging Electronic Markets, 2003, pp. 11-22.

[13] L. Bangyong, T. Jie, and L. Juanzi, "Association search in semantic web: Search+Inference," in International World Wide Web Conference, 2005.

[14] B. Aleman-Meza, C. Halaschek-Wiener, I. B. Arpinar, and A. Sheth, "Context-aware semantic association ranking," in First International Workshop on Semantic Web and Databases, Berlin, Germany, 2003, pp. 33-50.

[15] J. Heflin and J. Hendler, "Searching the web with SHOE," in AAAI-2000 Workshop on AI for Web Search, 2000.

[16] C. Rocha, D. Schwabe, and M. de Aragao, "A hybrid approach for searching in the semantic web," in Proceedings of the 13th international conference on World Wide Web, New York, NY, USA, 2004, pp. 374 383 .

[17] W. Buntine, K. Valtonen, and M. P. Taylor, "The ALVIS document model 
for a semantic search engine," in 2nd Annual European Semantic Web Conference, Heraklion, Crete, 2005.

[18] D. Bonino, F. Corno, and L. Farinetti, "DOSE: a distributed open semantic elaboration platform," in ICTAI 2003, The 15th IEEE International Conference on Tools with Artificial Intelligence, Sacramento, California, 2003.

[19] K. van der Sluijs, "Search the semantic web," Master's thesis, Department of Mathematics and Computer Science, Technical University of Eindhoven, 2004

[20] J. Robin and F. Ramalho, "Can ontologies improve web search engine effectiveness before the advent of the semantic web?" in SBBD 2003, Manaus, Brazil, 2003, pp. 157-169.

[21] H. Zhu, J. Zhong, J. Li, and Y. Yu, "An approach for semantic search by matching RDF graphs," in In Proceedings of the Special Track on Semantic Web at the 15th International FLAIRS Conference (sponsored by $A A A I)$, Florida, USA, 2002.

[22] V. Tamma, I. Blacoe, B. Smith, and M. Wooldridge, "SERSE: searching for semantic web content," in In Proceedings of the 16th European Conference on Artificial Intelligence, ECAI 2004, Valencia, Spain, 2004.

[23] P. Spyns, D. Oberle, R. Volz, J. Zheng, M. Jarrar, Y. Sure, R. Studer, and R. Meersman, "OntoWeb - A semantic web community portal," in Proceedings of the 4th International Conference on Practical Aspects of Knowledge Management, 2002, pp. 189 - 200.

[24] J. Contreras, V. R. Benjamins, M. Blzquez, S. Losada, R. Salla, J. Sevilla, D. Navarro, J. Casillas, A. Momp, D. Patn, L. Rodrigo, P. Tena, and I. Martos, "International Affairs Portal: A semantic web application," in ECAI Workshop on Application of Semantic Web Technologies to Web Communities, 2004.

[25] A. Sheth, C. Bertram, D. Avant, B. Hammond, K. Kochut, and Y. Warke, "Managing semantic content for the web," IEEE Internet Computing, vol. 6(4), pp. $80-87$, Sep 2002.

[26] M. Biddulph, "Crawling the semantic web," in XML Europe 2004, Netherlands, 2004.

[27] C. Patel, K. Supekar, Y. Lee, and E. Park, "Ontokhoj: A semantic web portal for ontology searching, ranking and classification," in Proceedings of ACM Fifth International Workshop on Web Information and Data Management (WIDM), New Orleans, 2003, pp. 58-61.

[28] W. Nejdl, "How to build Google2Google - An (incomplete) recipe," in 3rd International Semantic Web Conference, Hiroshima, Japan, 2004.

[29] van Hage Willem, M. de Rijke, and M. Marx, "Information retrieval support for ontology construction and use," in Proceedings 3rd International Semantic Web Conference (ISWC 2004), 2004.

[30] R. Baeza-Yates and B. Ribeiro-Neto, Modern Information Retrieval. Addison-Wesley, 1999. 